\title{
Hypogelsolinemia and Decrease in Blood Plasma Sphingosine-1-Phosphate in Patients Diagnosed with Severe Acute Pancreatitis
}

\author{
Tomasz Wollny ${ }^{1}$ Marzena Wątek ${ }^{1,2}$ - Urszula Wnorowska ${ }^{3}$. Ewelina Piktel ${ }^{3}$. Stanisław Góźdź ${ }^{1,4}$ • Krzysztof Kurek ${ }^{5}$. \\ Przemysław Wolak ${ }^{4} \cdot$ Grzegorz Król $^{4} \cdot$ Małgorzata Żendzian-Piotrowska ${ }^{6} \cdot$ Robert Bucki $^{3}$ (i)
}

Received: 25 April 2020 / Accepted: 20 January 2021 / Published online: 23 February 2021

(c) The Author(s) 2021

\begin{abstract}
Background Acute pancreatitis (AP) is a frequent hospitalization cause of patients suffering from gastrointestinal disorders. Gelsolin has an ability to bind bioactive lipids including different sphingolipids engaged in inflammatory response. Importantly, hypogelsolinemia was observed in patients with different states of acute and chronic inflammation.

Aims The aim of the present study was to assess the interplay of blood plasma gelsolin and blood plasma sphingosine1-phosphate (S1P) concentration in patients diagnosed with acute pancreatitis.

Materials and Methods To assess the concentration of gelsolin and S1P, immunoblotting and HPLC technique were employed, respectively. Additionally, the concentrations of amylase, lipase, C-reactive protein (CRP), procalcitonin (PCT) and the number of white blood cells (WBC) and platelet (PLT) were recorded.

Results We found that both pGSN and S1P concentrations in the plasma of the AP patients were significantly lower (pGSN $\sim 15-165 \mathrm{mg} / \mathrm{L} ; \mathrm{S} 1 \mathrm{P} \sim 100-360 \mathrm{pmol} / \mathrm{mL}$ ) when compared to the levels of pGSN and S1P in a control group (pGSN 130-240 mg/L; S1P 260-400 pmol/mL). Additionally, higher concentrations of CRP, WBC, amylase and lipase were associated with low level of gelsolin in the blood of AP patients. No correlations between the level of PCT and PLT with gelsolin concentration were noticed.

Conclusion Plasma gelsolin and S1P levels decrease during severe acute pancreatitis. Simultaneous assessment of pGSN and S1P can be useful in development of more accurate diagnostic strategies for patients with severe acute pancreatitis.
\end{abstract}

Keywords Gelsolin $\cdot$ Sphingosine-1-phosphate $\cdot$ Acute pancreatitis

\section{Introduction}

Acute pancreatitis (AP), an inflammatory disorder of the pancreas, is one of the leading hospital admission causes when providing care for patients with gastrointestinal disorders in the Western world [1]. In case of severe AP, a

Robert Bucki

buckirobert@gmail.com

1 Holy Cross Oncology Center of Kielce, Artwińskiego 3, 25-734 Kielce, Poland

2 Department of Hematology, Institute of Hematology and Transfusion Medicine, Indiry Gandhi 14, 02-776 Warsaw, Poland

3 Department of Medical Microbiology and Nanobiomedical Engineering, Medical University of Białystok, Mickiewicza 2C, 15-222 Białystok, Poland mortality rate is ranging from 36 to $50 \%$. Gallstones and alcohol abuse are long-established risk factors; however, several new causes have emerged, together with new aspects of pathophysiology that improved our understanding of AP disorder. Recent studies showed the new molecular mechanisms and pathways leading to severe AP development;

4 Institute of Medical Science, Collegium Medicum, Jan Kochanowski University of Kielce, IX Wieków Kielc 19A, 25-317 Kielce, Poland

5 Department of Gastroenterology and Internal Medicine, Medical University of Bialystok, Skłodowskiej-Curie 24A, 15-276 Bialystok, Poland

6 Department of Hygiene, Epidemiology and Ergonomics, Medical University of Bialystok, Mickiewicza 2C, 15-222 Bialystok, Poland 
however, successful treatment of AP has not yet been established [1]. AP diagnosis is made based on compatible clinical features including abdominal pain, nausea, and vomiting. Moreover, the clinical suspicion of the disease is supported by the finding of serum amylase elevations and/or lipase levels increase; most recommendations and most expert opinions suggest a level of at least three times the upper limit of normal as the most accurate cutoff [2]. A very important issue is the classification of severity of AP, as appeared for the first time in the proceedings of an international symposium held in Atlanta, Georgia, in September 1992 [3]. Then, it has been revised; severe acute pancreatitis was defined as the presence of organ failure and/or local pancreatic complications, complemented by the presence of unfavorable prognostic signs (using Ranson's criteria or Acute Physiology and Chronic Health Evaluation [APACHE] II). The definition included both criteria that predict development of severe disease [3].

Recent studies underlined the role of sphingolipids (SL) in the pathophysiology of AP $[4,5]$. Initially known for their role as cellular membrane building molecules or as metabolic substrates [6], their function as a source of signaling mediators and receptor agonists is now established. Sphingolipid-delivered mediators are synthesized through de novo pathway or may origin from the hydrolysis of complex lipids, mainly sphingomyelin, that is present in the plasma membranes of all human cells [7]. The list of most important lipids of the sphingolipid signaling cascade includes ceramide (CER), ceramide-1-phosphate (C1P), sphingosine (SPH), and sphingosine-1-phosphate (S1P). S1P was firstly described as a mediator of embryonic development, primarily cardiogenesis, angiogenesis, and vasculogenesis [8, 9]. Currently, attention is focused on its role in cardiovascular and neurological disorders, tumor biology, inflammation, and lymphocyte trafficking $[10,11]$. The role of SL and their metabolites in the regulation of cell proliferation and apoptosis was also recently underlined in many studies [12-14]. Additionally, SL might interact with extracellular proteins such as plasma gelsolin that was first discovered as an intracellular actin-binding protein involved in the remodeling of cellular actin filaments associated with cell shape changes and movement [15]. Extracellular gelsolin that is present in the blood (200-300 mg/L) has been described as the part of extracellular actin scavenging system and is also involved in the presentation of lysophosphatidic acid and other inflammatory mediators to their receptors $[16,17]$. It was shown that plasma gelsolin level decreases during acute injury and inflammation in a variety of clinical conditions such as acute respiratory distress syndrome, sepsis, major trauma, prolonged hyperoxia, malaria, liver injury and rheumatoid arthritis $[16,18,19]$. In the present study, we described the changes in plasma gelsolin and S1P in blood collected from patients diagnosed with severe AP.

\section{Materials and Methods}

\section{Subjects}

The investigation was approved by the Ethical Committee for Human Studies of the Medical University of Bialystok. The enrollment to the study took place between August 2015 and November 2019 in the Department of Gastroenterology and Internal Medicine (Medical University of Bialystok). One hundred and forty-four patients with AP hospitalized in the Department of Gastroenterology and Internal Medicine (Medical University of Białystok) were screened for inclusion. We have predicted severe acute pancreatitis based on APACHE II score. Patients with persistent (more than $48 \mathrm{~h}$ ) organ failure have been classified as the severe form of the disease; 38 subjects were qualified. Among them, one patient had a history of relapsing acute pancreatitis and one patient died before the end of the study protocol. Finally, 36 subjects were included for further examination. The size of research sample was not calculated, because it was exploratory study. The inclusion criteria were: 1) an individual age greater than 18 years old, 2) absence of any pancreatic disorders in the past (based on the hospital records and interview), 3) a confirmed diagnosis of AP (the presence of at least two out of three following criteria: (1) typical abdominal pain, (2) more than a threefold increase in serum lipase or amylase activity, (3) typical AP lesions observed in CT, MR or USG.

The exclusion criteria were: (1) lack of patients written consent, (2) onset of symptoms more than $24 \mathrm{~h}$ before admission, and (3) previous history of any pancreatic disease. The degree of AP severity was established in accordance with the revision of the Atlanta classification [20]. All patients (Table 1) received standard AP treatment, intensive intravenous hydration with lactated Ringer' solution in the initial phase of the disease and pain management. Endoscopic revision of biliary ducts was performed on patients with confirmed biliary etiology, within $24 \mathrm{~h}$ after admission. The patients also received early enteral feeding. The ones with persistent multiorgan dysfunction were transferred to intensive care units. The control group consisted of healthy volunteers, similar to the studied group with respect to sex and age. They were professionally active, did not have serious chronic diseases, and were admitted to work by an occupational medicine doctor.

\section{Serum Analyses}

Blood samples were taken from peripheral vein into heparinized syringe in the first day after onset of AP 
Table 1 Demographic characteristics of the patients included in the study. Number of patients, their age, sex, and etiology of AP

\begin{tabular}{ll}
\hline Variable & Value \\
\hline Number of patients diagnosed with pancreatitis & $n=36$ \\
Age (years) & \\
Mean $( \pm$ SD) & $46 \pm 15.28$ \\
Range & $21-78$ \\
Sex, number & \\
Male & $19(53 \%)$ \\
Female & $17(47 \%)$ \\
Acute pancreatitis etiology: & \\
Alcohol abuse & $n=22(61 \%)$ \\
Biliary & $n=12(33 \%)$ \\
Hypertriglyceridemia & $n=2(6 \%)$ \\
Frequency of organ failure & \\
Multiple organ failure & $10(28 \%)$ \\
Pulmonary failure & $21(58 \%)$ \\
Renal failure & $9(25 \%)$ \\
Hepatic failure & $4(11 \%)$ \\
Cardiovascular failure & $8(22 \%)$ \\
Control & $n=36$ \\
Age (years) & \\
Mean ( \pm SD) & $42 \pm 17.3$ \\
Range & $23-68$ \\
Sex, number $(\%)$ & $17(47 \%)$ \\
Male & $19(53 \%)$ \\
Female & \\
\hline
\end{tabular}

symptoms. Samples were centrifuged and the procured plasma was frozen in $-80^{\circ} \mathrm{C}$ until further analyses. A set of commercially available kits (Abbot, USA) was used to measure serum amylase, lipase, alanine aminotransferase (ALT), aspartate aminotransferase (AST) activity, serum CRP, PCT, bilirubin, fibrinogen, and creatinine contents.

\section{Quantitative Immunoblotting of Plasma Gelsolin}

Plasma samples were boiled in the presence of sample buffer for $5 \mathrm{~min}$ at $95{ }^{\circ} \mathrm{C}$ and subjected to electrophoresis on $10 \%$ sodium dodecyl sulfate (SDS)-polyacrylamide. Samples loaded in each gel were accompanied by recombinant human plasma gelsolin (rhp-GSN) standard (loaded in a concentration range comparable to the gelsolin concentration in the samples). After electrophoresis, proteins were transferred to polyvinylidene difluoride (PVDF) membrane (Immobilon ${ }^{\circledR}-F L$ PVDF, Millipore). The membrane was then blocked by incubation in Odyssey blocking buffer (LiCor). Transferred proteins were probed with a monoclonal antihuman gelsolin antibody (Sigma, St. Louis, MO, USA) used at a 1:10,000 dilution in TBS-T. After incubation with secondary goat anti-mouse IgG $(\mathrm{H}+\mathrm{L})$ IRDye $800 \mathrm{CW}$
(1:20,000, Li-Cor), immunoblots were visualized using the Li-Cor Odyssey ${ }^{\circledR}$ scanner and an Image Studio ${ }^{\mathrm{TM}}$ software (Li-Cor Biosciences). The intensity of each band on the membrane minus the background signal was plotted versus the known amount of gelsolin and fitted to a straight line $\left(R^{2} \geq 0.9\right)[21]$.

\section{Evaluation of S1P in Blood Plasma}

The sphingosine-1-phosphate concentration was measured by the method described in [22]. Briefly, acidified methanol and an internal standard ( 30 pmol of C17-S1P, Avanti Polar Lipids) were added to $250 \mu \mathrm{L}$ of plasma or CSF and then the samples were ultrasonicated in ice-cold water for $1 \mathrm{~min}$. The lipids were then extracted with chloroform, $1 \mathrm{M} \mathrm{NaCl}$, and $3 \mathrm{~N} \mathrm{NaOH}$. The alkaline aqueous phase containing $\mathrm{S} 1 \mathrm{P}$ was transferred to a fresh tube. Residual S1P in the chloroform phase was re-extracted twice with a methanol/1 M $\mathrm{NaCl}(1: 1, \mathrm{v} / \mathrm{v})$ solution, and then all aqueous fractions were combined. The amount of S1P was determined indirectly after dephosphorylation to sphingosine using alkaline phosphatase (bovine intestinal mucosa, Fluka, Milwaukee, WA). To improve the extraction yield of released sphingosine, chloroform was carefully placed at the bottom of the reaction tubes. The chloroform fraction containing dephosphorylated sphingoid base was washed three times with alkaline water ( $\mathrm{pH}$ adjusted to 10.0 with ammonium hydroxide) and then evaporated under a nitrogen stream. The dried lipid residues were redissolved in ethanol, converted to their o-phthalaldehyde derivatives, and analyzed using an HPLC system (ProStar, Varian Inc.) equipped with a fluorescence detector and a C18 reversed-phase column (Varian Inc. OmniSpher 5, $4.6150 \mathrm{~mm}$ ). The isocratic eluent composition of acetonitrile (Merck):water $(9: 1, \mathrm{v} / \mathrm{v})$ and a flow rate of $1 \mathrm{ml} / \mathrm{min}$ were used. The column temperature was maintained at $33{ }^{\circ} \mathrm{C}$ by use of a column oven.

\section{Statistical Analysis}

Data are reported as a mean \pm SD. Statistical significance was determined using two-tailed Student's $t$ test. Statistical analyses were performed using OriginPro 9.65 (OriginLab, Northampton, MA, USA). $* * * P \leq 0.001$.

\section{Results}

\section{Decrease in Gelsolin and Sphingosine-1-Phosphate Concentrations in the Course of Severe Acute Pancreatitis}

We observed a significant reduction in the concentration of pGSN in the blood of patients with AP compared to the control 


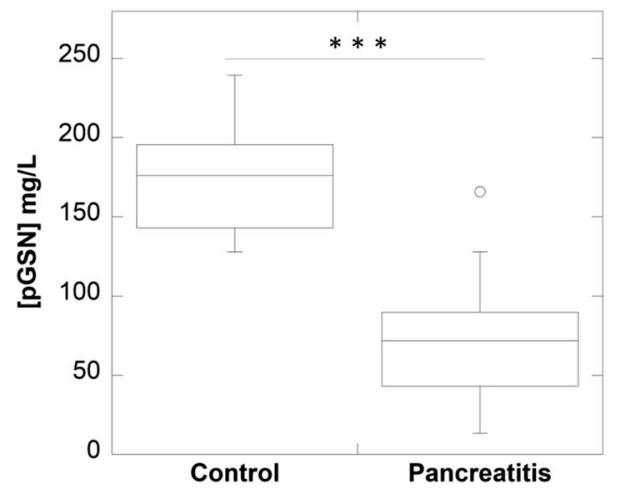

Fig. 1 Gelsolin concentration in plasma samples collected from control volunteers $(n=36)$ and patients diagnosed with severe acute pancreatitis $(n=36)$ during first day of diagnosis. From all study participants, blood samples were taken using heparin as an anticoagulant. Student's $t$ test was used to confirm statistical differences between two sets of data $(* * * P \leq 0.001)$ when compared to control

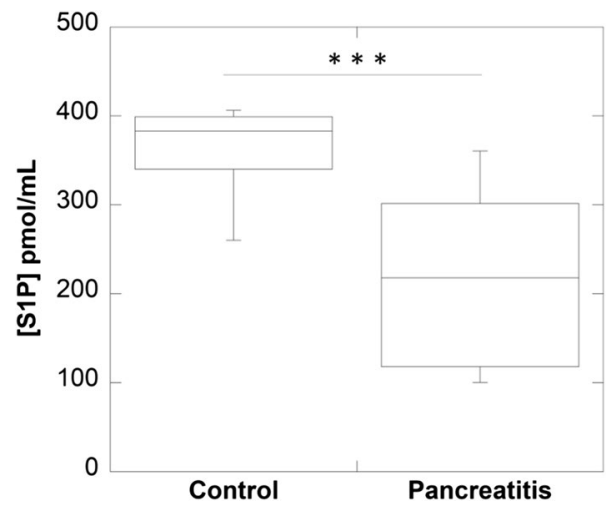

Fig. 2 Decrease in sphingosine-1-phosphate concentrations (pmol/ $\mathrm{mL}$ ) in the course of severe acute pancreatitis. Student's $t$ test was used to confirm statistical differences between two sets of data $(* * * P \leq 0.001)$ when compared to control

group (Fig. 1). pGSN level in control group was ranging between 127 and $239 \mathrm{mg} / \mathrm{L}$, and in the patients diagnosed with acute pancreatitis pGSN concentration was ranging between 14 and $165 \mathrm{mg} / \mathrm{L}$. Similar trends were obtained when S1P blood levels were measured (Fig. 2). Concentration of S1P was much higher $(260-400 \mathrm{pmol} / \mathrm{mL})$ in a control group compared to patients diagnosed with AP $(100-360 \mathrm{pmol} / \mathrm{mL})$. The obtained results confirm that plasma gelsolin and S1P levels decrease in the result of inflammation that developed during severe acute pancreatitis.

\section{Correlations of pGSN Concentration with Other Diagnostic Markers of AP}

While looking for new diagnostic tools to better describe the condition of patients with AP, we compared CRP, WBC, amylase, lipase, PCT and PLT levels with the blood pGSN levels (Fig. 3). We observed the positive correlation of pGSN levels with AP-associated factors in four cases (Fig. 3A-D). Increased serum $\mathrm{C}$-reactive protein level was associated with low pGSN concentrations; $R=0.74$ (Fig. $3 \mathrm{~A}$ ), which confirmed that concentrations of CRP rise in response to inflammation and it is important to determine its value during AP diagnosis. Similar results were observed in relation to the count of white blood cells; $R=0.61$ (Fig. 3B). Levels of serum amylase or serum lipase are increased during AP, and our results confirmed that low concentration of gelsolin correlates with high level of amylase and lipase; $R=0.70$ and $R=0.62$, respectively (Fig. 3C, D). Correlation between count of PLT, concentration of PCT, and concentration of plasma gelsolin was not observed; $R=0.15, R=0.08$, respectively (Fig. 3E, F).

\section{Correlations of pGSN Concentration with Other Biochemical Markers}

The markers assessing liver and kidney function (AST, ALT, serum bilirubin, fibrinogen, and creatinine) were elevated in some patients in severe AP. However, as shown in Fig. 4, these biochemical markers levels were not correlated with plasma gelsolin concentration (as shown in Fig. 4: for AST: $R=0.10$, ALT: $R=0.26$, bilirubin: $R=0.02$, fibrinogen: $R=0.06$, creatinine: $R=0.09$ ).

\section{Discussion}

The severe form of AP comprising about $20-30 \%$ of the patients is a life-threatening disease with the mortality rates of about 15\% [23]. Severe acute pancreatitis develops in about $25 \%$ of patients with AP and is often associated with single or multiple organ dysfunction requiring intensive care. Nowadays, some trends in the management of severe acute pancreatitis have changed our clinical practices. Between them, early enteral feeding, avoiding surgery in patients with sterile necrosis, more conservative approach to infected necrosis with delayed intervention, whether endoscopic or surgical, and management of biliary pancreatitis are worth to be mentioned [24]. In the early stages, the treatment for AP is largely supportive and reactive. Delays in the identification of early organ dysfunction are associated with a higher risk of morbidity and mortality [25]. However, the successful dealing with the AP patients and the management of severe acute pancreatitis are hampered by the limited understanding of pathogenesis and multicausality of the AP, uncertainties to predict outcome and a few effective treatment modalities. Therefore, comprehensive explanation of the pathophysiological and immunological mechanisms of AP, investigation into 
different biochemical pathways and search for early biomarkers of the AP severity, is a pressing issue.

It is known that gelsolin and S1P are both among an extremely small group of proteins and lipids that function in both extracellular and intracellular compartments. In the extracellular environment, plasma gelsolin functions in part as an actin scavenger, while S1P acts as an agonist for numerous cell types; their strong involvement in inflammatory processes is well documented [26]. Activation of sphingosine kinase 1 (SphK1) by a variety of cytokines and concomitant formation of S1P are important for various inflammatory responses [27], it is also likely that gelsolin-S1P interaction may control several steps of inflammation.

In this work, we aim to examine the connections between plasma gelsolin- and SL-delivered mediators (S1P) in the course of early stage of severe AP. We have observed significant decrease in both gelsolin and S1P concentrations in studied AP patients. We speculate that there is a close connection between significant reduction of gelsolin level and decrease in S1P concentration noticed in our patients. Moreover, hypogelsolinemia was correlated with the blood amylase and lipase activity as well as CRP concentration, suggesting that hypogelsolinemia might be an essential pathophysiological event leading to severe AP. It is worth to underline that both plasma gelsolin and S1P may play a role by regulating endothelial barrier function $[28,29]$. Plasma gelsolin is known to prevent negative consequences of circulating F-actin released from damage cells toward endothelial cells forming microvessels; on the other hand, S1P is well-established factor that enhances vascular barrier function acting as an agonist of sphingosine 1-phosphate receptor $1\left(\mathrm{~S}_{1} \mathrm{P}_{1}\right)$ with following downstream triggering of the Rho family of small GTPases, reorganization of cell cytoskeletal, focal adhesion formation, and tight junction assembly [30-32].

Literature data suggested that SL signaling pathway is altered in many gastrointestinal disorders [5, 33, 34]. As shown previously, a marked decrease in S1P concentration reflects AP severity [5]. It has been also suggested, using some animal models, that S1P plays a significant role in development of the inflammation; inhibition of S1P synthesis diminished the inflammatory response in septic mice [35], while sphingosine kinase-depleted animals showed a lower local and systemic inflammation response in the dextran sulfate sodium-induced inflammatory bowel disease (IBD) [36].

Previous clinical studies demonstrated that plasma gelsolin depletion precedes and therefore might predict secondary inflammation and tissue injury; authors suggested that hypogelsolinemia should be recognized as an indicator of critical complication or/and poor prognosis [16, 37].
Interesting observations regarding gelsolin functions have recently come from the vertebrate model organisms [38]. This study provides evidence that gelsolin was not only a crucial protein during development, but rather in processes involving rapid and dynamic actin cytoskeleton rearrangement, such as inflammatory stress responses. However, as authors emphasize, since gelsolin has more than one mechanism of action, its role might be different case by case; on the one hand, it can be seen as an inflammatory cytokine, on the other hand: a sharp decrease in its concentration can lead to an increase in mortality. In animal sepsis in vivo, administration of rhu-pGSN subcutaneously to mice and rats challenged with $E$. coli lipopolysaccharide endotoxin or subjected to cecal ligation-puncture significantly reduced their mortality in the absence of antibiotics [39, 40]. Improvement in clinical outcome was also observed upon intravenous administration of rhu-pGSN in mice exposed to $P$. aeruginosa challenge [41].

$\mathrm{Li}$ and coworkers in the group of 22 patients with severe AP showed that S1P measured in peripheral neutrophils and lymphocytes was markedly elevated in the early stage of disease [4]. These data are not consistent with our results. The explanation for this phenomenon may be related to the fact that platelets and erythrocytes, being the main source of S1P in the blood, could release it into the plasma in a agonistdependent manner [42], while the mechanisms regulating SL release from other blood cells are still not fully elucidated. Moreover, it has been demonstrated that both S1P and its analogue (FTY 720) application could reduce pulmonary inflammations and injuries in rats with experimental AP [43], and the others showed that FTY720 contributes in a reduction of the severity of pancreatic necrosis [44].

Actually, it is hard to conclude whether S1P-gelsolin interaction is good or harmful in the course of severe AP. We can speculate that gelsolin may impair S1P/S1P-receptor system that changes lymphocyte distribution within lymphoid organs and egress to the blood and involves S1Pmediated modulation of endothelial cell barrier function, in particular endothelial stiffness $[45,46]$. Therefore, S1P-gelsolin complexes (that decrease in AP) might be important in regulating endothelial permeability and function as the prevention tools of fluid accumulation in tissues. Such process might have a great importance in AP patients, indicating a likely therapeutic benefit of gelsolin-mediated S1P functional interplay that might be potentially restored upon recombinant human plasma gelsolin administration, and further clinical research is needed to prove this hypothesis.

Observation of Kononczuk et al. [5] is consistent with our results; they have also found significant reduction of SL metabolites, including S1P, in early stages of severe AP. Interestingly, sphingosine-1-phosphate concentration was not changed in patients with mild or moderate AP. Moreover, the concentration of S1P returned to normal values 
Digestive Diseases and Sciences (2022) 67:536-545

541
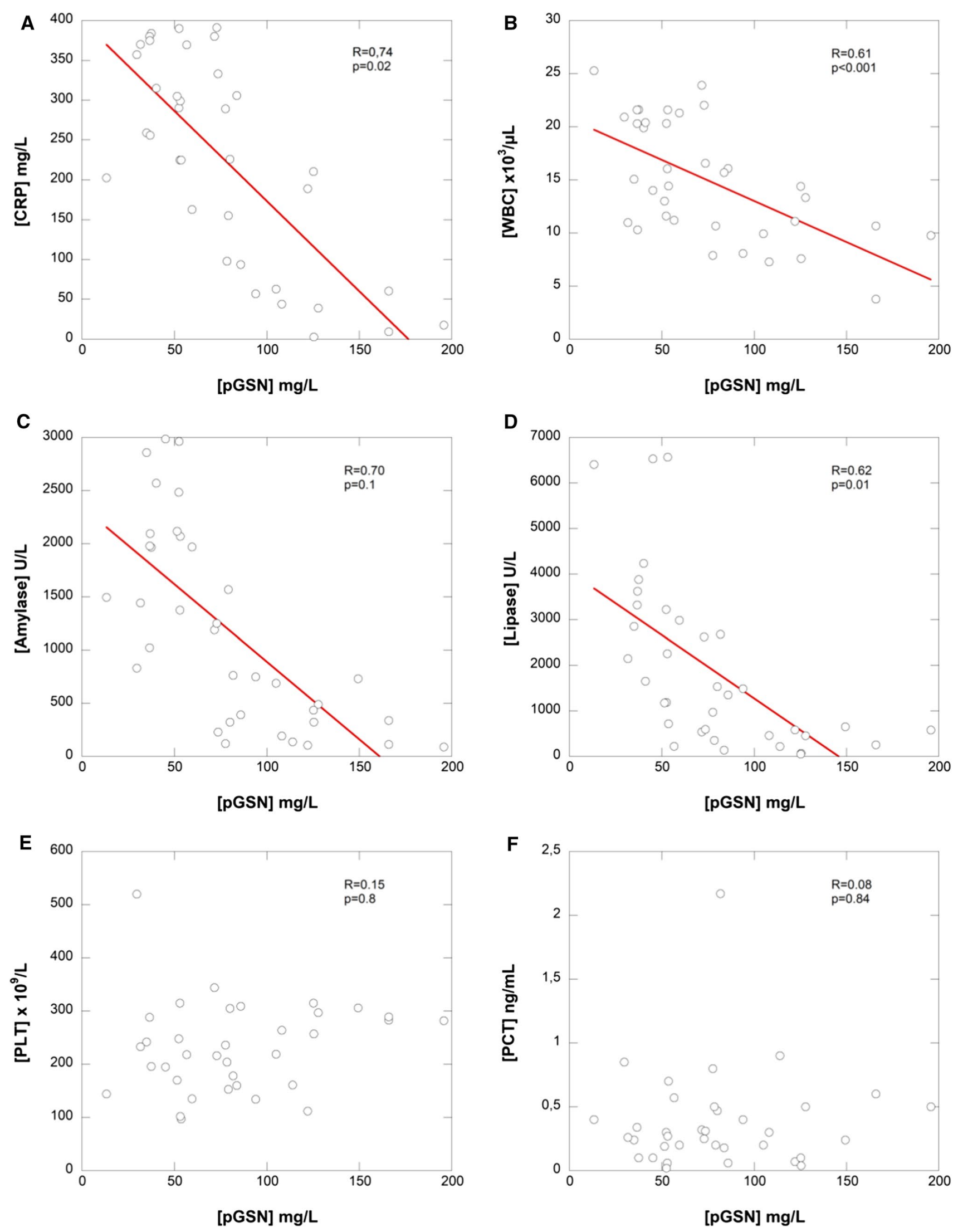

Springer 
4Fig. 3 Correlation plot of plasma gelsolin versus CRP (A), WBC $(\mathbf{B})$, amylase (C), lipase (D), PCT (E) and PLT (F) concentration in blood plasma collected from subjects included in the acute pancreatitis patient group. $R^{2}$ values were calculated using a linear curve fit. $P<0.05$ was considered to be statistically significant

after 7 days. In our opinion, our founding support mentioned above observation.

We postulate that S1P and plasma gelsolin measurement in early stages of AP may play an important role as biomarkers in accurate AP diagnosis and patient's risk stratification. Moreover, data obtained from animal studies justify the need for further research on gelsolin supplementation in patients suffering from life-threatening conditions such as severe acute pancreatitis. However, further clinical studies are needed to justify such a possibility.

\section{Conclusion}

Decrease in plasma gelsolin and S1P levels during severe acute pancreatitis might provide an opportunity for future development of new diagnostic and therapeutic strategies to assess and improved clinical stage of patients suffering from this disease. However, due to preliminary nature of our founding, further studies are needed to confirm such a possibility. 

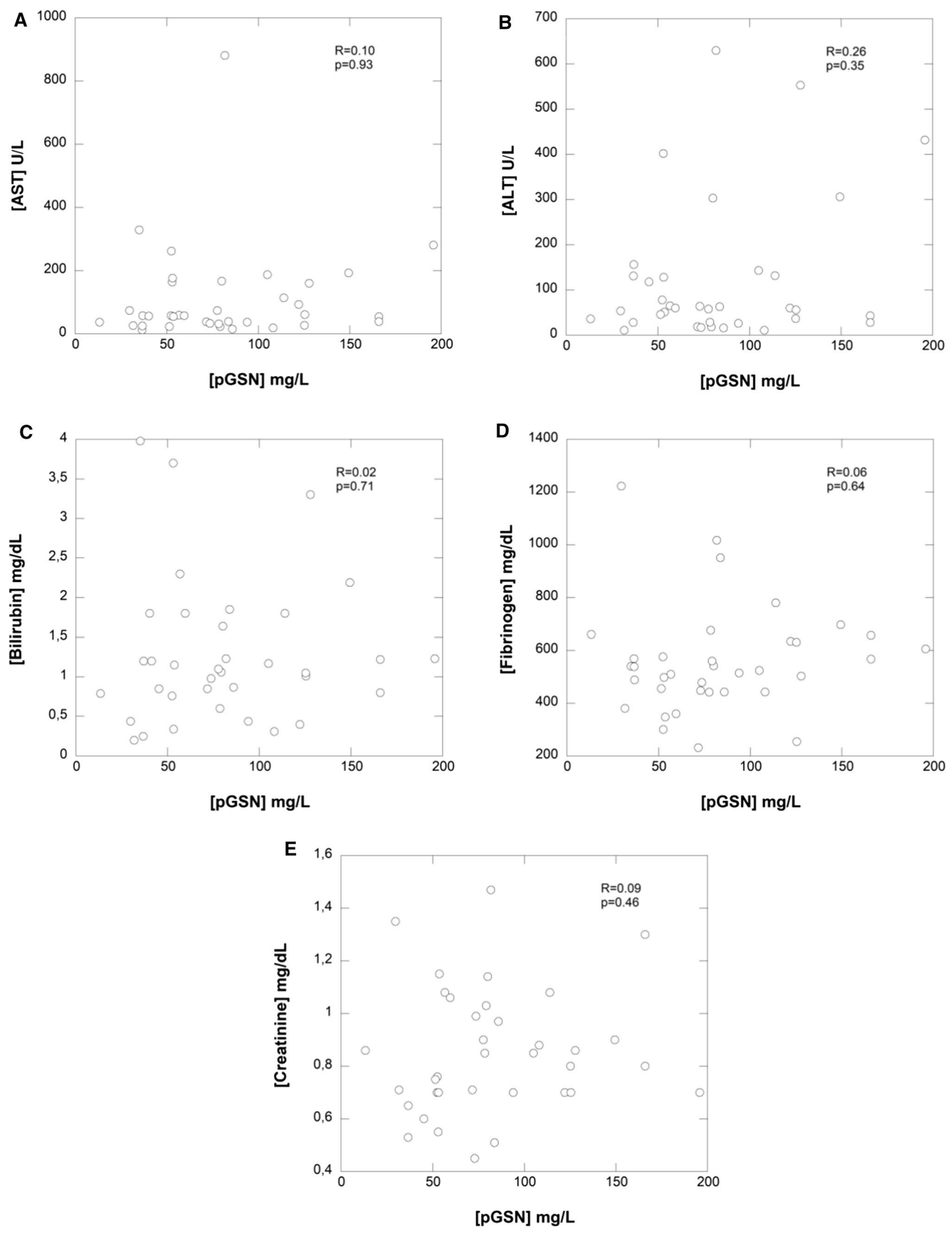
४Fig. 4 Correlation plot of plasma gelsolin versus aspartate aminotransferase (AST) (A), alanine aminotransferase (ALT) (B), bilirubin $(\mathbf{C})$, fibrinogen $(\mathbf{D})$, and creatinine $(\mathbf{E})$ concentration in blood plasma collected from subjects included in the acute pancreatitis patients group. $R$ ' values were calculated using linear curve fit. $P<0.05$ was considered to be statistically significant

Acknowledgments This work was supported by the National Science Center, Poland, under Grant: UMO-2015/17/B/NZ6/03473 (to RB).

\section{Compliance with Ethical Standards}

Conflict of interest The authors declare that they have no conflict of interest.

Open Access This article is licensed under a Creative Commons Attribution-NonCommercial 4.0 International License, which permits any non-commercial use, sharing, adaptation, distribution and reproduction in any medium or format, as long as you give appropriate credit to the original author(s) and the source, provide a link to the Creative Commons licence, and indicate if changes were made. The images or other third party material in this article are included in the article's Creative Commons licence, unless indicated otherwise in a credit line to the material. If material is not included in the article's Creative Commons licence and your intended use is not permitted by statutory regulation or exceeds the permitted use, you will need to obtain permission directly from the copyright holder. To view a copy of this licence, visit http://creativecommons.org/licenses/by-nc/4.0/.

\section{References}

1. Lankisch PG, Apte M, Banks PA. Acute pancreatitis. Lancet 2015;386:85-96.

2. Forsmark CE, Baillie J. AGA Institute technical review on acute pancreatitis. Rev Gastroenterol Mex 2007;72:257-285.

3. Bradley EL, 3rd. A clinically based classification system for acute pancreatitis. Summary of the International Symposium on Acute Pancreatitis, Atlanta, GA, September 11 through 13, 1992. Arch Surg. 1993;128:586-590.

4. Li Q, Wang C, Zhang Q, Tang C, Li N, Li J. The role of sphingosine kinase 1 in patients with severe acute pancreatitis. Ann Surg 2012;255:954-962.

5. Kononczuk T, Lukaszuk B, Zendzian-Piotrowska M et al. Plasma sphingolipids in acute pancreatitis. Int J Mol Sci 2017;18:2606.

6. Divecha N, Irvine RF. Phospholipid signaling. Cell 1995;80:269-278.

7. Bartke N, Hannun YA. Bioactive sphingolipids: metabolism and function. J Lipid Res 2009;50:S91-96.

8. Liu Y, Wada R, Yamashita T et al. Edg-1, the G protein-coupled receptor for sphingosine-1-phosphate, is essential for vascular maturation. J Clin Investig 2000;106:951-961.

9. Watek M, Piktel E, Wollny T et al. Defective sphingolipids metabolism and tumor associated macrophages as the possible links between gaucher disease and blood cancer development. Int J Mol Sci. 2019;20:843.

10. Sabbadini RA. Targeting sphingosine-1-phosphate for cancer therapy. Br J Cancer 2006;95:1131-1135.

11. Rivera J, Proia RL, Olivera A. The alliance of sphingosine1-phosphate and its receptors in immunity. Nat Rev Immunol 2008;8:753-763.
12. Burns TA, Luberto C. Sphingolipid metabolism and leukemia: a potential for novel therapeutic approaches. Anti-Cancer Agents Med Chem 2011;11:863-881.

13. Maceyka M, Payne SG, Milstien S, Spiegel S. Sphingosine kinase, sphingosine-1-phosphate, and apoptosis. Biochim et Biophys Acta 2002;1585:193-201.

14. Ogretmen B, Hannun YA. Biologically active sphingolipids in cancer pathogenesis and treatment. Nat Rev Cancer 2004;4:604-616.

15. Yin HL, Stossel TP. Control of cytoplasmic actin gel-sol transformation by gelsolin, a calcium-dependent regulatory protein. Nature 1979;281:583-586.

16. Bucki R, Levental I, Kulakowska A, Janmey PA. Plasma gelsolin: function, prognostic value, and potential therapeutic use. Curr Prot Pept Sci 2008;9:541-551.

17. Piktel E, Levental I, Durnas B, Janmey PA, Bucki R. Plasma gelsolin: indicator of inflammation and its potential as a diagnostic tool and therapeutic target. Int J Mol Sci 2018;19:2516.

18. Osborn TM, Verdrengh M, Stossel TP, Tarkowski A, Bokarewa M. Decreased levels of the gelsolin plasma isoform in patients with rheumatoid arthritis. Arthritis Res Ther 2008;10:R117.

19. Watek M, Wnorowska U, Wollny $\mathrm{T}$ et al. Hypogelsolinemia in patients diagnosed with acute myeloid leukemia at initial stage of sepsis. Med Sci Monit 2019;25:1452-1458.

20. Banks PA, Bollen TL, Dervenis C et al. Classification of acute pancreatitis--2012: revision of the Atlanta classification and definitions by international consensus. Gut 2013;62:102-111.

21. Mounzer KC, Moncure M, Smith YR, Dinubile MJ. Relationship of admission plasma gelsolin levels to clinical outcomes in patients after major trauma. Am J Respir Crit Care Med 1999;160:1673-1681.

22. Min JK, Yoo HS, Lee EY, Lee WJ, Lee YM. Simultaneous quantitative analysis of sphingoid base 1-phosphates in biological samples by o-phthalaldehyde precolumn derivatization after dephosphorylation with alkaline phosphatase. Anal Biochem 2002;303:167-175.

23. van Santvoort HC, Bakker OJ, Bollen TL et al. A conservative and minimally invasive approach to necrotizing pancreatitis improves outcome. Gastroenterology 2011;141:1254-1263.

24. Leppaniemi A, Tolonen M, Tarasconi A et al. WSES guidelines for the management of severe acute pancreatitis. World J Emerg Surg 2019;2019:27.

25. Anand N, Park JH, Wu BU. Modern management of acute pancreatitis. Gastroenterol Clin N Am 2012;41:1-8.

26. Bucki R, Kulakowska A, Byfield FJ et al. Plasma gelsolin modulates cellular response to sphingosine 1-phosphate. Am J Physiol Cell Physiol. 2010;299:C1516-C1523.

27. Chalfant CE, Spiegel S. Sphingosine 1-phosphate and ceramide 1-phosphate: expanding roles in cell signaling. J Cell Sci 2005;118:4605-4612.

28. Xiong YQ, Hla T. S1P control of endothelial integrity. Curr Top Microbiol 2014;378:85-105.

29. Stepanovska B, Lange AI, Schwalm S, Pfeilschifter J, Coldewey SM, Huwiler A. Downregulation of S1P lyase improves barrier function in human cerebral microvascular endothelial cells following an inflammatory challenge. Int J Mol Sci 2020;21:1240.

30. Wang LC, Dudek SM. Regulation of vascular permeability by sphingosine 1-phosphate. Microvasc Res 2009;77:39-45.

31. Burg N, Swendeman S, Worgall S, Hla T, Salmon JE. Sphingosine 1-phosphate receptor 1 signaling maintains endothelial cell barrier function and protects against immune complex-induced vascular injury. Arthritis Rheumatol. 2018;70:1879-1889.

32. Miura Y, Yatomi Y, Rile G, Ohmori T, Satoh K, Ozaki Y. Rhomediated phosphorylation of focal adhesion kinase and myosin light chain in human endothelial cells stimulated with sphingosine 
1-phosphate, a bioactive lysophospholipid released from activated platelets. J Biochem 2000;127:909-914.

33. Kurek K, Lukaszuk B, Piotrowska DM, Wiesiolek P, Chabowska AM, Zendzian-Piotrowska M. Metabolism, physiological role, and clinical implications of sphingolipids in gastrointestinal tract. Biomed Res Int 2013;2013:908907.

34. Wollny T, Wątek M, Durnaś B et al. Sphingosine-1-phosphate metabolism and its role in the development of inflammatory bowel disease. Int J Mol Sci 2017; 18:741.

35. Puneet $\mathrm{P}$, Yap CT, Wong L et al. SphK1 regulates proinflammatory responses associated with endotoxin and polymicrobial sepsis. Science 2010;328:1290-1294.

36. Snider AJ, Kawamori T, Bradshaw SG et al. A role for sphingosine kinase 1 in dextran sulfate sodium-induced colitis. FASEB $J$ 2009;23:143-152.

37. Dinubile MJ. Plasma gelsolin: in search of its raison d'etre. Focus on "Modifications of cellular responses to lysophosphatidic acid and platelet-activating factor by plasma gelsolin." Am J Physiol Cell Physiol 2007;292:C1240-C1242.

38. Migocka-Patrzalek M, Niedbalska-Tarnowska J, Garbiec A, Dubinska-Magiera M, Daczewska M. Gelsolin and related proteins in vertebrate model organisms. Folia Biol-Krakow 2019;67:159-167.

39. Cohen TS, Bucki R, Byfield FJ et al. Therapeutic potential of plasma gelsolin administration in a rat model of sepsis. Cytokine 2011;54:235-238.
40. Lee PS, Waxman AB, Cotich KL, Chung SW, Perrella MA, Stossel TP. Plasma gelsolin is a marker and therapeutic agent in animal sepsis. Crit Care Med 2007;35:849-855.

41. Piktel E, Wnorowska U, Cieśluk $\mathrm{M}$ et al. Recombinant human plasma gelsolin stimulates phagocytosis while diminishing excessive inflammatory responses in mice with Pseudomomas aeruginosa sepsis. Int J Mol Sci 2020;21:2551.

42. Ito K, Anada Y, Tani M et al. Lack of sphingosine 1-phosphatedegrading enzymes in erythrocytes. Biochem Biophys Res Commun 2007;357:212-217.

43. Liu J, Xu P, Zhang L et al. FTY720 attenuates acute pancreatitis in hypertriglyceridemic apolipoprotein CIII transgenic mice. Shock 2015;44:280-286.

44. Muller CA, Belyaev O, Burr W et al. Effects of FTY720 and rapamycin on inflammation in taurocholate-induced acute pancreatitis in the rat. Pancreas 2012;41:1086-1091.

45. Gollmann G, Neuwirt H, Tripp CH et al. Sphingosine-1-phosphate receptor type-1 agonism impairs blood dendritic cell chemotaxis and skin dendritic cell migration to lymph nodes under inflammatory conditions. Int Immunol. 2008;20:911-923.

46. Rosen H, Sanna G, Alfonso C. Egress: a receptor-regulated step in lymphocyte trafficking. Immunol Rev 2003;195:160-177.

Publisher's Note Springer Nature remains neutral with regard to jurisdictional claims in published maps and institutional affiliations. 